\title{
Stress and velocity fields in glaciers: Part I. Finite-difference schemes for higher-order glacier models
}

\author{
Jacques Colinge, ${ }^{1}$ Heinz Blatter ${ }^{2}$ \\ 1 Département de Mathématiques, Université de Genève, CH-1211 Genève 24, Switzerland \\ ${ }^{2}$ Geographisches Institut, Eidgenössische Technische Hochschule, CH-8057 Zürich, Switzerland
}

\begin{abstract}
The set of force equations and stress-strain-rate relations for ice masses can be solved with the method of lines and shooting the stress-free conditions at the free surface. Single- and multiple-shooting schemes with fixed point or Newton iterations for solving the stress equations including the deviatoric stress gradients are described and their performances are discussed. The single-shooting Newton iteration proved to be the fastest scheme for typical valley glaciers, although its horizontal grid limitation is restrictive. Grid resolution can be improved substantially with a multiple-shooting scheme but computation time and storage requirements increase substantially. The Newton iteration allows the handling of mixed basal boundary conditions, partly basal velocity and partly basal shear traction being prescribed. A stick-slip free gravity flow illustrates the performance of the scheme.
\end{abstract}

\section{INTRODUCTION}

Several studies have approached questions on flow patterns in ice sheets near the summit (Reeh, 1988; Dahl-Jensen, 1989) and the transition from ice-sheet flow to ice-shelf flow (Herterich, 1987) by including the role of deviatoric stress gradients. Van der Veen and Whillans (1989) and Van der Veen (1989) have presented a numerical scheme that solves the equations of force balance without any mathematical approximations. They start their iteration from a "first approximation" that formally solves the equations of force balance in a form today referred to the shallow-ice approximation and improve on this approximate solution. Their fixed-point iteration scheme seems to work efficiently for ice sheets, though no account is given about its performance in the case of smaller and steeper glaciers. Muller (1991) proposed a similar approach for solving the first-order equations for stress and velocity components in the twodimensional plane-flow approximation. This algorithm, based on finite-difference discretization along the $x$ axis (method of lines) and a fixed-point iteration scheme to meet the surface-boundary conditions, proved to be very simple and flexible, and could be readily extended to the threedimensional case (Blatter, 1995).

The fixed-point iteration applied by Muller (1991) and Blatter (1995) converges rapidly for large ice-sheet configurations with a small aspect ratio. However, for small glaciers, where corrective terms become important, the number of necessary iteration steps grows rapidly with the increasing aspect ratio. Furthermore, the number of necessary iteration steps also increases with increasing horizontal grid resolution. These factors limit the applicability of fixedpoint iteration in the achievable horizontal grid resolution and exclude small and steep glaciers, for which convergence cannot be achieved at all. The fixed-point iteration scheme can handle prescribed basal velocity and it can handle mixed basal boundary conditions, prescribed basal velocity in some parts and basal shear traction in other parts of the bed. However, with mixed boundary conditions, convergence of the iteration is so poor that fixed-point iteration in practice is restricted to prescribing the basal velocity along the entire bed. However, this procedure then suffers from obvious deficiencies of adequate imposition of boundary conditions.

In this paper, an improved algorithm is presented. The replacement of fixed-point iteration by a Newton iteration scheme substantially reduces the number of necessary iteration steps. Although a single Newton iteration step requires more computations, the total computation time is considerably reduced. Furthermore, the new technique easily allows the imposition of mixed basal boundary conditions. The limitation in the horizontal grid resolution can be reduced by replacing the single- by a multiple-shooting scheme. With this multiple-shooting scheme, the convergence criterion is no longer the limiting factor in many applications. However, the large memory required for handling the linear algebra limits its applicability.

The problem treated in this paper does not handle the complete glacier-modelling problem. The acceleration term can be omitted in the momentum-balance equations, though not, as sometimes stated, because acceleration is negligibly small (Whillans, 1987). On the contrary, acceleration is large enough that the time needed to adjust the velocity field to a new situation is negligibly short (seconds) compared with the time-scales that are relevant for glaciological studies (hours to years). Therefore, stress and velocity fields are treated as quasi-stationary, and that has an important consequence. The stress and velocity fields only depend on the instantaneous state of the geometry and other conditions that are relevant for the rheology but 
not their rates of changes. In this sense, the mechanical problem treated in section 2 constitutes a complete mathematical problem that can be solved independently of mass balance and consequent changes in geometry.

This paper presents the methods of multiple-shooting and Newton iteration schemes, and the handling of different types of basal boundary conditions. Here, we discuss the two-dimensional equations, even though the three-dimensional problem closely follows the same line as the simpler case. The mathematical details, important information on the implementation and performance of the routines are presented by Colinge (paper in preparation), together with practical examples. A practical application of the three-dimensional, first-order scheme on Haut Glacier d'Arolla has been presented by Hubbard and others (1998). The performance of multiple-shooting the Newton iteration scheme has been demonstrated by Blatter and others (1998). In this paper, referred to as Paper II in the remainder of this paper, the relation between basal stress distribution and basal movement is investigated in detail.

\section{STRESS AND VELOGITY FIELDS}

\section{Governing equations}

Here, a summary of the model equations in the plane-flow approximation is presented. A scale analysis is conducted that suggests a perturbation-type solution procedure of the governing equations in terms of a small parameter. Correspondingly, equations are called zeroth, first- and higherorder equations depending upon whether they contain the small parameter in the zeroth, first or higher powers. The second-order equations of the conservation of mass and momentum and the flow law (constitutive relationship) are listed and a detailed description of the integration scheme as well as the Newton iteration scheme for its solution in the two-dimensional plane-flow approximation are given.

The geometry of the ice mass is defined by the upper free surface $z=S(x)$ and the basal surface $z=B(x)$, in which $(x, z)$ are orthogonal Cartesian coordinates with the $z$ axis pointing opposite to the direction of gravity. As is common in glaciology, the strain-rate tensor $\mathbf{D}$ and the stress deviator $\boldsymbol{\Sigma}$ are related by

$$
\mathbf{D}=A F\left(I_{I I}\right) \boldsymbol{\Sigma}, \quad I_{I I}=\frac{1}{2} \operatorname{tr}\left(\boldsymbol{\Sigma}^{2}\right)
$$

where the rate factor $A$ is, in general, a function of the temperature but is held constant here where considerations are limited to isothermal conditions. The fluidity $F$ depends on the second invariant of the stress deviator, the traditional choice being $F\left(I_{I I}\right)=\left(I_{I I}\right)^{(n-1) / 2}$ with a proven law exponent $n \approx 3$. Regularity of the integration procedure requires a finite viscosity law, requesting $F(0) \neq 0$, different from Glen's flow law.

The total stress $\mathbf{T}$ will be decomposed into pressure $P$ and the stress deviator $\mathbf{\Sigma}, \mathbf{T}=-P \mathbf{1}+\Sigma$, normal components will be denoted by $\sigma_{k k}$ (no summation, $k=x, z$ ) and shear-stress components by $\tau_{i j}(i, j=x, z)$, while $\tau_{k k}$ are the total normal stress components.

To obtain an objective hierarchy of approximations, we scale the spatial variables $x, z$ and $S$, the horizontal and ver- tical velocity components $u$ and $w$, the stresses, the rate factor and the fluidity by

$$
\begin{aligned}
(x, z, S) & =\{L\}(\tilde{x}, \epsilon \tilde{z}, \epsilon \tilde{S}), \\
(u, w) & =\{A\}\{H\}(\rho g\{H\} \epsilon)^{n}(\tilde{u}, \epsilon \tilde{w}), \\
\left(P, \tau_{k k}, \tau_{i j}, \sigma_{k k}\right) & =\rho g\{H\}\left(\tilde{P}, \tilde{\tau}_{k k}, \epsilon \tilde{\tau}_{i j}, \epsilon \tilde{\sigma}_{k k}\right), \\
A & =\{A\} \tilde{A}, \\
F & =(\rho g H \epsilon)^{n-1} \tilde{F}
\end{aligned}
$$

where the tilded quantities are dimensionless and $\{\Phi\}$ is the dimensional order of magnitude for a dimensional quantity $\Phi$. Typical vertical and horizontal extents of the ice mass are $\{H\}$ and $\{L\}$, respectively, and the aspect ratio is $\epsilon=\{H\} /\{L\} ; \rho$ and $g$ are the constant density of ice and acceleration of gravity. The horizontal velocity component $u$ is scaled with the shear velocity in a homogencous parallel-sided slab of thickness $\{H\}$ when flow law in Equation (1) with its power-law fluidity, $\tilde{F}$, is imposed; the vertical velocity component $w$ is taken to be a factor $\epsilon$ smaller. Pressure and total normal stress, $P$ and $\tau_{k k}$ are scaled with the hydrostatic pressure at the bed of a parallel-sided slab, whilst shear stress $\tau_{i j}(i \neq j)$ and normal deviatoric stresses $\sigma_{k k}$ are scaled with the basal driving stress.

For glaciers, the aspect ratio $\epsilon$ is small and can be used as an order-of-magnitude estimate of the various terms in the mass- and force-balance equations and the constitutive equations. In plane flow, these laws take the form (see Blatter, 1995)

$$
\begin{gathered}
\frac{\partial \tilde{u}}{\partial \tilde{x}}+\frac{\partial \tilde{w}}{\partial \tilde{z}}=0, \\
2 \epsilon \frac{\partial \tilde{\sigma}_{x x}}{\partial \tilde{x}}+\frac{\partial \tilde{\tau}_{x z}}{\partial \tilde{z}}=\frac{\partial \tilde{S}}{\partial \tilde{x}}-\epsilon^{2} \underbrace{\frac{\partial^{2}}{\partial \tilde{x}^{2}}\left(\int_{\tilde{z}}^{\hat{S}} \tilde{\tau}_{x z} \mathrm{~d} z^{\prime}\right)}_{T}, \\
\epsilon \frac{\partial \tilde{u}}{\partial \tilde{x}}=\tilde{A} \tilde{F}\left(t^{2}\right) \tilde{\sigma}_{x x},
\end{gathered}
$$

in which for $n=3$

$$
\frac{\partial \tilde{u}}{\partial \tilde{z}}+\epsilon^{2} \frac{\partial \tilde{w}}{\partial \tilde{x}}=2 \tilde{A} \tilde{F}\left(t^{2}\right) \tilde{\tau}_{x z}
$$

$$
\begin{aligned}
F\left(t^{2}\right) & =t^{2}+t_{0}^{2} ; \\
t^{2} & \equiv \tilde{\tau}_{x z}^{2}+\tilde{\sigma}_{x x}^{2},
\end{aligned}
$$

and $t_{0}^{2}$ is proportional to the inverse of the viscosity at vanishing effective stress. Note that Equation (8) is obtained from the horizontal force balance by an integration over depth and incorporating the vertical force balance and free-surface stress-free boundary conditions (see e.g. Hutter, 1983, chapter 5).

We now apply a terrain following coordinate transformation by mapping the local ice thickness $h$ on to the unit interval

$$
\xi=\tilde{x}, \quad \zeta=\frac{\tilde{z}-\tilde{B}}{\tilde{S}-\tilde{B}} .
$$

We also employ the notation

$$
\begin{aligned}
\tilde{\tau}=\tilde{\tau}_{x z}, \quad \tilde{\sigma}=\tilde{\sigma}_{x x}, \quad \hat{\tau}=\tilde{\tau}-2 c \tilde{\sigma}, \\
a \equiv \frac{1}{h}\left[(1-\zeta) \frac{\partial^{2} \tilde{B}}{\partial \xi^{2}}+\zeta \frac{\partial^{2} \tilde{S}}{\partial \xi^{2}}\right], \\
c \equiv(1-\zeta) \frac{\partial \tilde{B}}{\partial \xi}+\zeta \frac{\partial \tilde{S}}{\partial \xi} \\
b=\frac{\partial c}{\partial \zeta}
\end{aligned}
$$


where $\hat{\tau}$ is the shear traction parallel to the surface defined by $\zeta=$ constant. In terms of the new independent variables, the transformed $T$ term takes the form

$$
T=\frac{\partial^{2} I}{\partial \xi^{2}}+\frac{c^{2}}{h^{2}} \frac{\partial^{2} I}{\partial \zeta^{2}}+2 \frac{c}{h} \frac{\partial^{2} I}{\partial \xi \partial \zeta}+\frac{2 c b-a h}{h^{2}} \frac{\partial I}{\partial \zeta}
$$

where

$$
I \equiv h \int_{\zeta}^{1} \tilde{\tau} \mathrm{d} \zeta^{\prime}
$$

With the above transformation rules, the field Equations (7)-(10) may be written in the form

$$
\begin{gathered}
\frac{\partial \tilde{w}}{\partial \zeta}=-\frac{\partial \tilde{u}}{\partial \xi} \\
\frac{\partial \hat{\tau}}{\partial \tilde{\zeta}}=-2 \epsilon b \tilde{\sigma}-h\left(2 \epsilon \frac{\partial \tilde{\sigma}}{\partial \xi}-\frac{\mathrm{d} \tilde{S}}{\mathrm{~d} \xi}\right)-\epsilon^{2} T \\
\frac{\partial \tilde{u}}{\partial \zeta}=-\epsilon^{2} h \frac{\partial \tilde{w}}{\partial \xi}+h \tilde{A} \tilde{F}(2 \hat{\tau}+3 c \epsilon \tilde{\sigma}), \\
0=\frac{\partial \tilde{u}}{\partial \xi}+c \frac{\partial \tilde{w}}{\partial \xi}-\tilde{A} \tilde{F}\left[2 c \hat{\tau}+\left(1+3 c^{2}\right) \tilde{\sigma}\right] .
\end{gathered}
$$

This is a set of four equations for the variables $\tilde{u}, \tilde{w}, \hat{\tau}$ and $\tilde{\sigma}$ and, if it were not for the $T$ term, it would be a system of first-order partial differential equations. This $T$ term makes it a system of integro-differential equations, which is of second order in the $\xi$ derivative. Solutions to it are sought in the domain bounded by the free and basal surfaces, for which boundary conditions need to be imposed. In the transformed terrain following coordinates, the locations of their imposition are $\zeta=0$ and $\zeta=1$.

Physically, the boundary condition at the free surface consists of a vanishing shear traction (vanishing horizontal air-pressure gradient is already incorporated in Equation (8))

$$
\hat{\tau}_{\mathrm{s}}=0 \text {. }
$$

At the basal surface, the form of the boundary conditions depends on the local conditions that are postulated to apply. Either one assumes no-slip conditions and then has

$$
\tilde{u}=\tilde{w}=0 \text { at } \zeta=0
$$

or a certain sliding law. In this latter case, the basal shear traction $\hat{\tau}_{\mathrm{b}}$ and the sliding velocity $\hat{u}_{\mathrm{b}}$ are functionally related via

$$
\mathcal{F}\left(\hat{\tau}_{\mathrm{b}}, \hat{u}_{\mathrm{b}}\right)=0
$$

but, in addition, the tangency condition of the flow must hold

$$
\tilde{w}_{\mathrm{b}}=\frac{\mathrm{d} \tilde{B}(x)}{\mathrm{d} \tilde{x}} \tilde{u}_{\mathrm{b}} .
$$

The simplest case is perfect slip for which $\hat{\tau}_{\mathrm{b}}=0$. It is obvious that a total of three boundary conditions must be imposed, either Equations (23) and (24) or else (23), (25) and (26).

These facts suggest the field Equations (19)-(22) should be regarded not as a system of partial differential equations but rather as though they were ordinary differential equations to be integrated in the $\zeta$ direction. (This will be formally achieved by replacing all $\xi$ derivatives by finite differences in the following section.) We have made this interpretation implicitly apparent in Equations (19)-(22) by writing the terms involving the $\zeta$ derivatives on the lefthand side and all other terms on the righthand side. Viewed this way, Equations (19)-(22) are three first-order ordinary differential equations (ODEs) in $\zeta$ and one algebraic equation for $u, w, \hat{\tau}$ and $\sigma$, the $\zeta$ integration of which indeed requires three boundary or initial conditions as surmised above. Because one condition is imposed at $\zeta=1$ and two are imposed at $\zeta=0$, i.e. at the two end points of the interval $\zeta \in[0,1]$, the problem is called a two-point boundary-value problem.

How this integration is performed will be explained below. Here, we simply mention that, as $\epsilon \rightarrow 0$, the above equations reduce to the zeroth-order equations, generally known as the shallow-ice approximation, which is commonly used in models of large ice sheets. For smaller mountain glaciers, the shallow-ice approximation misses significant physics. Deleting terms that contain $\epsilon^{2}$ yields the first-order approximation which already captures the essential patterns of the stress and velocity fields, and gives realistic results for small ice masses.

\section{Line integration}

The idea of discretizing the partial differential equations in every dimension except one to obtain a system of ordinary differential equations is called the method of lines (Verwer and Sanz-Serna, 1984). By introducing a discrete grid on the $\xi$ axis and approximating the $\xi$ derivatives by finite differences, Equations (19)-(21) can be rewritten as ODEs, and Equation (22) becomes algebraic. For each vertical gridline $i$, this establishes a set of three ODEs for the three unknowns $\hat{\tau}_{\mathrm{i}}, \tilde{u}_{\mathrm{i}}$ and $\tilde{w}_{\mathrm{i}}$ and one algebraic equation for $\tilde{\sigma}_{\mathrm{i}}$. This large set of ODEs can be integrated by using a standard numerical integrator (e.g. a Runge-Kutta scheme). The integration begins at the base $(\zeta=0)$, with starting values for $\hat{\tau}_{\mathrm{b}}$ and $\tilde{u}_{\mathrm{b}}$ at each gridpoint. At each step of the numerical integration, the algebraic equation is solved explicitly or with a numerical root-finder. It is important that the algebraic equation always has a unique real solution. This is the case for small aspect ratios generally relevant for glaciers and for the flow law in Equation (11) with a flow-law exponent $n=3$ (paper in preparation by J. Colinge).

To arrive at a proper finite-difference scheme, several points must be taken into account. The shear stress $\hat{\tau}$ is computed from Equation (20) and depends on $\partial \tilde{\sigma} / \partial \xi$ and $\tilde{\sigma}$. Furthermore, $\tilde{\sigma}$ is computed from Equation (22) and depends on $\partial \tilde{u} / \partial \xi$. This cascade of dependence on $\xi$ derivatives reduces the order of the whole difference scheme to $p-1$, even if all difference schemes for single derivatives are of the order $p$. Thus, to obtain a consistent difference scheme of order $p$, the $\partial \tilde{u} / \partial \xi$ in Equation (22) must be discretized to order $p+1$ (paper in preparation by J. Colinge).

The integration from $\zeta=0$ to $\zeta=1$ with the starting values for basal shear stress and velocity components, i.e. three conditions, does not automatically satisfy the surface boundary conditions in Equation (23). In order to solve the boundary-value problem, the proper basal values for $\hat{\tau}_{\mathrm{i}, \mathrm{b}}$ can be found iteratively. A good initial choice is the shallow-ice approximation of the basal shear stress, which in many cases is already close to the solution:

$$
\hat{\tau}_{\mathrm{i}, \mathrm{b}}^{0}=-h_{\mathrm{i}} \frac{\partial \tilde{S}_{\mathrm{i}}}{\partial \xi} .
$$

If basal conditions have a large variability, then an initial $\hat{\tau}_{\mathrm{i}, \mathrm{b}}^{0}=0$ is often the better choice to start with. With the basal shear stress and the values for the basal velocity com- 
ponents, Equation (22) is first used to calculate the basal value for $\tilde{\sigma}_{\mathrm{i}, \mathrm{b}}$. Integrating upwards from the base yields surface values $\hat{\tau}_{\mathrm{i}, \mathrm{s}}^{0} \neq 0$. By using this result, a correction to $\hat{\tau}_{\mathrm{i}, \mathrm{b}}^{0}$ must be found to obtain $\hat{\tau}_{\mathrm{i}, \mathrm{s}}^{1}$ closer to the required boundary condition. This method is called shooting, or single shooting (Ascher and others, 1988). The type of basal boundary conditions that must be met is defined by the sliding parameterization. Prescribing basal velocity is the simplest and numerically most stable case, except for the no-slip conditions of the limited value for studies of basal sliding. Mixed boundary conditions, vanishing basal velocity at non-sliding locations and basal friction represented by basal shear traction at sliding locations may be useful for specific studies on basal conditions. The application of a sliding law which relates basal shear traction to basal velocity requires a nested iteration of this sliding law within the shooting procedure.

The $T$ term (see Equation (17)) on the righthand side of Equation (20) requires different treatment. This term contains the integral of $\hat{\tau}$ and, because it is of the second order in the $\xi$ derivatives, it can only be computed after the line integration has been completed. In practice, the $T$ term is computed by using the $\hat{\tau}$ from the previous iteration step and then including it in Equation (20). Its inclusion makes the computation time per iteration step slightly longer but the number of necessary iteration steps is hardly affected. Numerical experiments show that the convergence becomes more difficult if nested iterations such as for sliding laws or for the $T$ term must be considered.

\section{Single-shooting fixed-point iteration}

A simple way to define a single-shooting procedure is to write the problem, Equations (20)-(22), as a fixed-point problem (Muller, 1991; Blatter, 1995). We introduce the following notation, where $M$ is the number of gridpoints for the method of lines:

$$
\begin{aligned}
\hat{T}_{\mathrm{b}} & =\left(\hat{\tau}_{1, \mathrm{~b}}, \cdots, \hat{\tau}_{M-1, \mathrm{~b}}\right)^{T}, \\
\hat{T}_{\mathrm{s}} & =\left(\hat{\tau}_{1, \mathrm{~s}}, \cdots, \hat{\tau}_{M-1, \mathrm{~s}}\right)^{T}, \\
G\left(\hat{T}_{\mathrm{b}}\right) & =\hat{T}_{\mathrm{b}}-\alpha \hat{T}_{\mathrm{s}}\left(\hat{T}_{\mathrm{b}}\right) .
\end{aligned}
$$

A converging fixed-point iteration is equivalent to a constructive proof of Banach's fixed-point theorem (Ascher and others, 1988). Thus, for $(\alpha>0, \alpha$ sufficiently small $)$ the fixed point $\hat{T}_{\mathrm{b}}^{*}$ of $G, G\left(\hat{T}_{\mathrm{b}}^{*}\right)=\hat{T}_{\mathrm{b}}^{*}$, is the correct solution.

In the simplified case of an infinite slab with a parallel flat surface and base, a simple criterion

$$
\alpha<4 e^{-8 / \Delta \xi}
$$

can be rigorously proven (paper in preparation by $\mathrm{J}$. Colinge) to ensure the existence and uniqueness of the desired fixed point (Banach's theorem). This exact criterion for the slab is approximately valid for the case of realistic glacier geometries and one can expect to be forced to decrease $\alpha$ either when $\Delta \xi$ decreases or the aspect ratio $\epsilon$ increases (see also Blatter, 1995).

This criterion is very restrictive and explains the impossibility of applying the method to small and steep glaciers. Moreover, it uses global quantities of ice geometry and local conditions may require a smaller $\alpha$ to achieve convergence. This is especially true if the surface slope locally displays large longitudinal variations, such as in icefalls or at steep glacier snouts. This criterion provides information regarding the stability of the numerical process. An unstable pro- cess is one which assigns largely different results to pairs of very close starting conditions. Since the idea of single shooting is to use the final results to learn something about the starting conditions, it is essential to avoid instability.

The restrictive criterion in Equation (31) also indicates the impossibility of applying the line integration in a single-shot inverse mode. One may be tempted to start the integration at the surface with zero shear traction and measured velocity components to compute basal shear traction and basal velocity (Van der Veen and Whillans, 1989). Although the mathematical problem is well posed, it is illconditioned and thus basal conditions obtained by this integration are extremely sensitive to the input at the surface and may be far away from the correct solution (see e.g. Lliboutry, 1987; Bahr and others, 1994).

\section{Single-shooting Newton iteration}

From the above consideration, we look for $\hat{T}_{\mathrm{b}}$ such that $\hat{T}_{\mathrm{s}}\left(\hat{T}_{\mathrm{b}}\right)=0$. Colinge (paper in preparation) has shown that $\hat{T}_{\mathrm{s}}$ is infinitely differentiable with respect to $\hat{T}_{\mathrm{b}}$. This is only true if the flow law $\tilde{F}$ is differentiable and invertible in the entire domain; otherwise, the uniqueness of the solution is no longer guaranteed, although the numerical integration may produce a result. Thus, Glen's flow law needs to be modified to avoid infinite viscosity for stress-free conditions (Hutter, 1981, 1983; Smith and Morland, 1981).

Another way of implementing a single-shooting procedure is by solving $\hat{T}_{\mathrm{s}}\left(\hat{T}_{\mathrm{b}}\right)=0$ with a Newton iteration:

$$
\hat{T}_{\mathrm{s}}\left(\hat{T}_{\mathrm{b}}\right)=\left(\begin{array}{c}
\hat{\tau}_{1, \mathrm{~s}}\left(\hat{\tau}_{1, \mathrm{~b}}, \cdots, \hat{\tau}_{M-1, \mathrm{~b}}\right) \\
\vdots \\
\hat{\tau}_{M-1, \mathrm{~s}}\left(\hat{\tau}_{1, \mathrm{~b}}, \cdots, \hat{\tau}_{M-1, \mathrm{~b}}\right)
\end{array}\right) .
$$

Then

$$
\hat{T}_{\mathrm{s}}\left(\hat{T}_{\mathrm{b}}+\delta \hat{T}_{\mathrm{b}}\right)=\hat{T}_{\mathrm{s}}\left(\hat{T}_{\mathrm{b}}\right)+\hat{T}_{\mathrm{s}}^{\prime}\left(\hat{T}_{\mathrm{b}}\right) \cdot \delta \hat{T}_{\mathrm{b}}+\mathcal{O}\left(\delta \hat{T}_{\mathrm{b}}^{2}\right)=0
$$

is a series expansion of $\hat{T}_{\mathrm{s}}$, where

$$
\hat{T}_{\mathrm{s}}^{\prime}\left(\hat{T}_{\mathrm{b}}\right)=\frac{\partial\left(\hat{\tau}_{1, \mathrm{~s}}, \cdots, \hat{\tau}_{r m M-1, s}\right)}{\partial\left(\hat{\tau}_{1, \mathrm{~b}}, \cdots, \hat{\tau}_{r m M-1, b}\right)}=\left(\frac{\partial \hat{\tau}_{i, \mathrm{~s}}}{\partial \hat{\tau}_{j, \mathrm{~b}}}\right)
$$

is the Jacobian matrix. To define the Newton iteration, we need to truncate Equation (33) and solve the remaining linear equation

$$
\delta \hat{T}_{\mathrm{b}}=-\left(\hat{T}_{\mathrm{s}}^{\prime}\left(\hat{T}_{\mathrm{b}}\right)\right)^{-1} \cdot \hat{T}_{\mathrm{s}}\left(\hat{T}_{\mathrm{b}}\right)
$$

and thus find the basal $\hat{T}_{\mathrm{b}}^{\text {new }}$ for the next from the old iteration step

$$
\hat{T}_{\mathrm{b}}^{\text {new }}=\hat{T}_{\mathrm{b}}^{\text {old }}+\delta \hat{T}_{\mathrm{b}} .
$$

The Newton iteration uses all gridpoints at the surface to correct the stress values at each gridpoint at the base and converges quadratically close to the solution (Ascher and others, 1988). On the other hand, the fixed-point iteration only uses the gridpoints at the surface to correct the basal stress in the same vertical profile and converges linearly. To compute the components of the Jacobian

$$
\frac{\partial \hat{\tau}_{i, \mathrm{~s}}}{\partial \hat{\tau}_{j, \mathrm{~b}}} \approx \frac{\hat{T}_{\mathrm{s}}\left(\hat{T}_{\mathrm{b}}+\delta \hat{T} \cdot e_{j}\right)-\hat{T}_{\mathrm{s}}\left(\hat{T}_{\mathrm{b}}\right)}{\delta \hat{T}}, \quad i=1, \cdots, M-1
$$

where

$$
e_{j}=(0, \cdots, 0 \underbrace{, 1,}_{j} 0, \cdots, 0)^{T}
$$

we have to integrate the set of equations $M-1$ times, once 
for each choice of the indices $j=1, \cdots, M-1$. The small increment $\delta T$ in the corresponding basal shear stress should be chosen as small as possible to obtain accurate values of the Jacobian components. The smallest and best choice for $\delta T$ is the square root of the machine precision.

For large $M$, the above computation of the Jacobian may become very expensive in computing time, since we only get one column of the matrix per integration. However, there is a great potential for reducing the number of necessary integrations. For physical reasons, we can anticipate that the components of the Jacobian become smaller if the difference $|j-i|$ grows, thus the Jacobian is approximately a banded matrix. The Newton-Kantorovitch theorem (Ascher and others, 1988) ensures the convergence of the Newton iteration even with an approximate Jacobian, provided the approximation is close enough. This suggests that we assume the Jacobian is a $k$ diagonal matrix, where $k$ is odd, and then calculate the approximate components of the Jacobian by

$$
\begin{gathered}
\frac{\partial \hat{\tau}_{i, \mathrm{~s}}}{\partial \hat{\tau}_{j \mathrm{~b}}} \approx \frac{\hat{T}_{\mathrm{s}}\left(\hat{T}_{\mathrm{b}}+\delta \hat{T} \cdot\left(e_{l}+e_{l+k}+e_{l+2 k} \cdots\right)\right)-\hat{T}_{\mathrm{s}}\left(\hat{T}_{\mathrm{b}}\right)}{\delta \hat{T}}, \\
i=1, \cdots, M-1
\end{gathered}
$$

for $l=1, \cdots, k$ and $j=l, l+k, l+2 k, \cdots$. This enables us to compute $M / k$ columns of the Jacobian matrix at each integration and thus reduces the number of necessary integrations to $k$, independently of the gencrally much larger number of gridlines $M$. Moreover, the linear system, Equation (35), can be solved faster by using a banded matrix. The bandwidth $k$ must be chosen such that $k \Delta \xi \geq 5 h$, i.e. beyond the range of influence of basal perturbations.

When the convergence threshold is not reached with the first iteration step, we repeat the above procedure of computing the Jacobian and integrate the equations with an improved choice of the basal shear traction. Sometimes the Newton iteration can be continued by keeping the old Jacobian, although convergence of the iteration may become slower than with an updated Jacobian or may not be achieved at all.

It is also possible to compute a number $C_{\text {single, }}$ called the condition, which describes the stability of the process (Ascher and others, 1988). For a slab, this number is

$$
C_{\text {single }}=\mathcal{O}\left[\frac{1}{\Delta \xi^{2}} \exp \left(\frac{8}{\Delta \xi}\right)\right] .
$$

A large condition number indicates an unstable algorithm which is very sensitive to initial (basal) values. In many cases, we observed numerically that, for a chosen geometry (synthetic or realistic), $\Delta \xi$ could be chosen about half the size than for the fixed-point iteration. This is a clear improvement, interesting for many studies, but the spatial resolution is still limited.

\section{Multiple-shooting Newton iteration}

The computation becomes unstable as $\Delta \xi$ decreases. One possibility of stabilizing the iteration scheme is to divide the glacier into $N$ horizontal layers, applying the single shooting with Newton iterations to each layer and require that the final solution has no discontinuity. More precisely, with the starting shear stresses and velocities $\tilde{T}_{0, j}, \tilde{U}_{0, j}$, $j=1, \cdots, N \quad\left(U_{0.1}\right.$ is given $)$ and the final values
$\hat{T}_{1, j}\left(\hat{T}_{0, j}, \tilde{U}_{0, j}\right), \tilde{U}_{1, j}\left(\hat{T}_{0, j}, \tilde{U}_{0, j}\right)$ we have to solve the following problem

$$
\left(\begin{array}{c}
\hat{T}_{1,1}\left(\hat{T}_{0,1}\right)-\hat{T}_{0,2} \\
\tilde{U}_{1,1}\left(\hat{T}_{0,1}\right)-\tilde{U}_{0,2} \\
\hat{T}_{1,2}\left(\hat{T}_{0,2}, U_{0,2}\right)-\hat{T}_{0.3} \\
\tilde{U}_{1,2}\left(\hat{T}_{0,2}, \tilde{U}_{0,2}\right)-\tilde{U}_{0,3} \\
\vdots \\
\hat{T}_{1, N-1}\left(\hat{T}_{0, N-1}, \tilde{U}_{0, N-1}\right)-\hat{T}_{0, N} \\
\tilde{U}_{1, N-1}\left(\hat{T}_{0, N-1}, \tilde{U}_{0, N-1}\right)-\tilde{U}_{0, N} \\
\hat{T}_{1, N}\left(\hat{T}_{0, N}, \tilde{U}_{0, N}\right)
\end{array}\right)=0
$$

in terms of $\hat{T}_{0, j}, \tilde{U}_{0, j}$

This approach is called multiple shooting (Ascher and others, 1988) and it is possible to prove for the slab that

$$
C_{\text {multiple }}=\mathcal{O}\left[\frac{1}{\Delta \xi^{3}} \exp \left(\frac{8}{N \Delta \xi}\right)\right] .
$$

This is a clear improvement, since $C_{\text {multiple }}<C_{\text {single }}$ and the stability improves for increasing $N$. It is also possible to prove for a slab with a linear flow law that such a process converges to the unique solution of the partial differential equation (paper in preparation by J. Colinge).

Again, the analysis of the simplified case of the slab has been relevant in the general case. So, this algorithm theoretically allows for any spatial resolution but requires much more computing time and memory (if $\Delta \xi$ is halved, then $N$ must be doubled). The fact that the stability of the algorithm suffers, if $\Delta \xi$ decreases is a property of the method of lines discretization. The condition number of the ODE system is

$$
C_{\mathrm{ODE}}=\mathcal{O}\left(\frac{1}{\Delta \xi}\right)
$$

which obviously grows as $\Delta \xi$ becomes smaller.

\section{Mixed basal boundary conditions}

The above algorithms and analyses do not only apply for prescribed zero basal velocity; any reasonable basal velocity can be imposed. However, in some cases it may be preferable to prescribe the basal shear traction in a given area of the glacier instead of the basal velocity itself. The algorithm still works in this more general application with little modification.

Again, it is possible to give a fixed-point formulation of the problem

$$
G\left(Y_{\mathrm{b}}\right)=Y_{\mathrm{b}}-\alpha \hat{T}_{\mathrm{s}}\left(Y_{\mathrm{b}}\right),
$$

where $Y_{i, \mathrm{~b}}=\hat{\tau}_{i, \mathrm{~b}}$ if $i \in E$ where $(E$ denotes the domain in which basal shear traction is prescribed) and $Y_{i, \mathrm{~b}}=\tilde{u}_{i, \mathrm{~b}}$ if $i \notin E, T_{\mathrm{s}}=\left(\hat{\tau}_{1, \mathrm{~s}}, \cdots, \hat{\tau}_{M-1, \mathrm{~s}}\right)^{T}$. The iteration parameter $\alpha$ must be chosen differently for the two domains with either prescribed basal velocity or prescribed basal shear traction. However, the fixed-point iteration was found to converge very slowly and, thus, its application in the case of mixed basal boundary conditions is not practical.

The resolution with a Newton iteration is preferable and, because the problem for mixed basal boundary conditions is less stable, multiple shooting is necessary. The algorithm must be prepared to include $u_{i, \mathrm{~b}}$ as an unknown if $i \notin E$ and $\hat{\tau}_{i, \mathrm{~b}}$ if $i \in E$. This implies computing a mixed Jacobian matrix by considering partial derivatives with respect to $\tilde{u}_{i, \mathrm{~b}}$ if $i \in E$ and with respect to $\hat{\tau}_{i, \mathrm{~b}}$ if $i \notin E$. 
In the basal section, where shear traction is prescribed, the basal velocity becomes a solution of the computation. This fact poses an additional problem with the discretization scheme. Using, for example, a symmetric second-order difference for $\partial \tilde{u} / \partial \xi$ makes the $\tilde{\sigma}$ at odd gridpoints dependent on $\tilde{u}$ at even gridpoints and vice versa. Additionally, a symmetric second-order scheme for $\partial \tilde{\sigma} / \partial \xi$ transfers this uncoupling to the solution of $\hat{\tau}$.

This uncoupling is less effective as long as the velocity component $u$ is prescribed along the entire bed and solutions are acceptable as long as variations in the basal velocity are small. However, if basal shear traction $\hat{\tau}_{\mathrm{b}}$ is prescribed instead at parts of the bed, the decoupling produces solutions for even and odd gridpoints which oscillate with a wavelength of two gridcells.

There are several possibilities for removing part of the oscillation, for example, by smoothing the solution with a filter function. However, the meaning of such a smoothed solution is not clear. A variation of this method is to smooth the solution for the basal velocity $\tilde{u}_{\mathrm{b}}$ within the region of prescribed basal shear traction and then to recompute a solution by using the algorithm prescribing this $\tilde{u}_{\mathrm{b}}$ instead. This furnishes a well-defined numerical solution to an approximate problem and the well-posedness of the problem suggests that this solution should be close to the true one. In practice, however, this method was not satisfactory. After smoothing $u_{\mathrm{b}}$, the corresponding $\hat{\tau}_{\mathrm{b}}$ was not as close to the originally prescribed one as desirable.

A preferable approach is to avoid the symmetries that disconnect the odd and even points, for example, by using non-symmetric finite differences to approximate $\partial \tilde{u} / \partial \xi$. There are several ways to do this but some ways remove only part of the oscillation (the odd and even points become only weakly coupled). The following scheme removes the oscillation to a large degree and also maintains the consistency in the order (second order) of the whole difference scheme. A fourth-order symmetric scheme for

$$
\frac{\partial \tilde{u}}{\partial \xi}\left(\xi_{i}, \zeta\right) \simeq \frac{-\tilde{u}_{i+2}(\zeta)+8 \tilde{u}_{i+1}(\zeta)-8 \tilde{u}_{i-1}(\zeta)+\tilde{u}_{i-2}(\zeta)}{12 \Delta \xi}
$$

is first used to compute $\tilde{\sigma}_{c}\left(x_{i}, \zeta\right)$. The corresponding $\tilde{F}\left(\tilde{\sigma}_{i}(\zeta), \hat{\tau}_{i}(\zeta)\right)$ is then used to compute the value of $\tilde{u}_{i}(\zeta+\Delta \zeta)$. The value of $\hat{\tau}_{i}(\zeta+\Delta \zeta)$ is dependent on $\tilde{\sigma}_{i}$ as well as on $\partial \tilde{\sigma} / \partial \xi$. For $\tilde{\sigma}_{i}$ the above $\tilde{\sigma}_{c}\left(x_{i}, \zeta\right)$ is used but

$$
\frac{\partial \tilde{\sigma}}{\partial \xi}\left(\xi_{i}, \zeta\right) \simeq \frac{\tilde{\sigma}_{\text {left }, i+1}(\zeta)-\tilde{\sigma}_{\text {right }, i-1}(\zeta)}{\Delta \xi}
$$

where $\tilde{\sigma}_{\text {left } i+1}(\zeta)$ is recomputed from Equation (22) with the asymmetric order-three scheme

$$
\frac{\partial \tilde{u}}{\partial \xi}\left(\xi_{i+1}, \zeta\right) \simeq \frac{2 \tilde{u}_{i+2}(\zeta)+3 \tilde{u}_{i+1}(\zeta)-6 \tilde{u}_{i}(\zeta)+u_{i-1}(\zeta)}{6 \Delta \xi}
$$

and $\tilde{\sigma}_{\text {right }, i-1}(\zeta)$ with

$$
\frac{\partial \tilde{u}}{\partial \xi}\left(\xi_{i-1}, \zeta\right) \simeq \frac{-\tilde{u}_{i+1}(\zeta)+6 \tilde{u}_{i}(\zeta)-3 \tilde{u}_{i-1}(\zeta)-2 \tilde{u}_{i-2}(\zeta)}{\Delta \xi} .
$$

This makes three separate computations of $\partial \tilde{u} / \partial \xi$ and solutions of Equation (22) necessary. On the other hand, this scheme has a well-defined order and meets the boundary conditions correctly. If Equation (22) is solved iteratively with a numerical root finder, the first solution, e.g. for $\tilde{\sigma}_{\mathrm{c}}$ is a very close approximation for the other two solutions $\tilde{\sigma}_{\text {left }}$ and $\tilde{\sigma}_{\text {right }}$, and thus allows a substantial reduction in the number of iteration steps. Using the average $\tilde{\sigma}_{\mathrm{c}}=\left(\tilde{\sigma}_{\text {right }}+\tilde{\sigma}_{\text {left }}\right) / 2$ produces the same solution (within the precision of the numerical scheme) as using the above suggested $\tilde{\sigma}_{\mathrm{c}}$.

\section{FLOW IN A PARALLEL-SIDED PLANE SLAB}

\section{Second- and first-order slab equations}

The geometry of the ice slab is defined by the upper free surface $S \equiv H$ and the basal surface $B \equiv 0$ in Cartesian coordinates $(x, z)$, with the $x$ axis aligned parallel to the direction of the surface slope. The $z$ axis and the direction of gravity include an angle $\alpha$. The equations are written in dimensionless form using the scaling

$$
\begin{aligned}
(\tau, \sigma) & =\rho g H \sin \alpha(\tilde{\tau}, \tilde{\sigma}), \quad i \neq j \\
u, w & =A_{0} H(\rho g H \sin \alpha)^{3}(\tilde{u}, \tilde{w}), \\
(x, z, S) & =H(\tilde{x}, \tilde{z}, \tilde{S}), \\
A & =A_{0} \tilde{A}, \\
F & =(\rho g H \sin \alpha)^{2} \tilde{F}
\end{aligned}
$$

where $\tau, \sigma, u$ and $w$ are the shear stress, deviatoric normal stress, longitudinal and transverse (vertical) velocity component, respectively. The symbols with the "tilde" are the corresponding dimensionless quantities; the rate factor $A$ is scaled with $A_{0}$ and Glen's exponent $n=3$.

The above scaling serves the purpose of writing the equations in dimensionless form and, thus, does not contain the scaling factor $\epsilon$. However, in the following equations we formally re-introduce factors $\epsilon$ and $\epsilon^{2}$ with $\epsilon=1$ to mark the first- and second-order terms corresponding to the scaling in Equations (2)-(6).

The dimensionless, two-dimensional second-order slab equations for the four unknowns $\tilde{\tau}, \tilde{\sigma}, \tilde{u}$ and $\tilde{w}$ are

$$
\begin{gathered}
\frac{\partial \tilde{w}}{\partial \tilde{z}}=-\frac{\partial \tilde{u}}{\partial \tilde{x}} \\
\frac{\partial \tilde{\tau}}{\partial \tilde{z}}=-2 \epsilon \frac{\partial \tilde{\sigma}}{\partial \tilde{x}}-1-\epsilon^{2} \frac{\partial^{2}}{\partial \tilde{x}^{2}}\left(\int_{\tilde{z}}^{1} \tilde{\tau} \mathrm{d} z^{\prime}\right), \\
\frac{\partial \tilde{u}}{\partial \tilde{z}}=-\epsilon^{2} \frac{\partial \tilde{w}}{\partial \tilde{x}}+2 A_{0} \tilde{F} \tilde{\tau} \\
0=\frac{\partial \tilde{u}}{\partial \tilde{x}}-\epsilon A_{0} \tilde{F} \tilde{\sigma}
\end{gathered}
$$

with the flow law in Equation (11) and the surface-boundary condition $\tilde{\tau}_{\mathrm{s}}=0$.

In the first-order approximation, Equations (55) and (56) reduce to

$$
\begin{gathered}
\frac{\partial \tilde{\tau}}{\partial \tilde{z}}=-2 \epsilon \frac{\partial \tilde{\sigma}}{\partial \tilde{x}}-1, \\
\frac{\partial \tilde{u}}{\partial \tilde{z}}=2 A_{0} \tilde{F} \tilde{\tau}
\end{gathered}
$$

whereas Equations (57) and (54) remain as in the secondorder form. Note that in the first-order approximation, the solution for $w$ from Equation (54) does not feed back to the solutions of the other three equations; thus, $w$ can be determined by quadrature of Equation (54) after the other three variables are computed.

The foregoing scaling reduces the number of free parameters for a homogeneous slab to two, namely $\tilde{t}_{0}^{2}$ and $A_{0}$. 
The rate factor $A_{0}$ is simply a multiplier for the velocity field and, as long as $A_{0}$ is homogeneous over the whole domain, the stress field is independent of $A_{0}$. This permits normalization of the velocity field for different choices of $\tilde{t}_{0}^{2}$ and makes $A_{0}$ a function of $\tilde{t}_{0}^{2}$, reducing the number of free parameters to the single parameter $\tilde{t}_{0}^{2}$. In all examples, we choose $\hat{t}_{0}^{2}$, and $A_{0}$ such that the asymptotic surface velocity

$$
\tilde{u}_{\mathrm{s}, \infty}=A_{0}\left(\frac{1}{2}+\tilde{t}_{0}^{2}\right)=1 .
$$

The asymptotic stress and velocity profiles are defined as the solution for the entirely non-sliding slab, i.e. the solution in the absence of any sliding perturbations. The limit of $A_{0} \rightarrow 0$ constitutes a Newton fluid, whereas the limit $\tilde{t}_{0}^{-2} \rightarrow 0$ constitutes the classical Glen flow law with infinite viscosity for vanishing effective stress. In all presented numerical examples, we choose $\tilde{t}_{0}^{2}=0.1$ and $A_{0}=5 / 3$. With this assumption, the only freedom rests in the choice of the basal stress pattern over a chosen area and/or the velocity over the remaining areas.

To simulate an infinitely long plane-strain slab, the model domain of finite length is numerically connected end to end. With homogeneous basal boundary conditions in the finite domain, this produces a homogeneous solution for the infinite slab; with inhomogeneous boundary conditions, this yields a periodic pattern with a wavelength of the length of the finite domain.

\section{Comparison between first- and second-order solu- tions}

The differences between first- and second-order equations are terms that contain derivatives of $\tilde{\tau}$ and $\tilde{w}$ with respect to $\tilde{x}$. These terms may become significantly large if sharp longitudinal transitions occur, either abrupt changes in the surface of bottom topography or sliding-non-sliding transitions. Only the latter are possible for parallel-sided ice slabs.

We have performed a number of computations with the first- and second-order models for a long non-sliding slab with a shorter zone of perfect slip. One representative example is illustrated in Figures $1-6$. The chosen grid size is $\Delta \tilde{x}=1.0$ and the length of the zone with frictionless sliding is defined by seven gridpoints with prescribed vanishing basal shear traction, $\tilde{\tau}_{\mathrm{b}}=0$, and the no-slip condition is imposed in the rest of the slab by $\tilde{u}_{\mathrm{b}}=0$. The transition occurs over one gridcell, i.e. over the distance of one slab thickness. The non-dimensional basal shear traction scales to $\tilde{\tau}_{\mathrm{b}}=1$ for non-sliding conditions far away from the influence of the sliding zone (asymptotic behaviour) and the corresponding shear velocity is normalized to $\tilde{u}_{\mathrm{s}}=1$.

In all cases, the basal shear traction reaches a peak value at the non-sliding gridpoint adjacent to the sliding zone and then drops sharply to the prescribed zero shear traction at the first sliding gridpoint (Fig. 1). Correspondingly, the longitudinal velocity component increases sharply, also changing from a typical shear-flow profile to nearly plough-flow in the interior of the sliding area (Figs 2 and 3). The transitions are also accompanied by a peak in downward vertical velocity where the ice enters sliding, and upwards, where ice leaves the sliding area (Fig. 4). Of course, the surface of the slab would change corresponding to the non-zero vertical velocity component and given surface mass flux. In this study, we do not investigate surface response to variations in basal conditions but we look at snapshots of the quasi-sta-

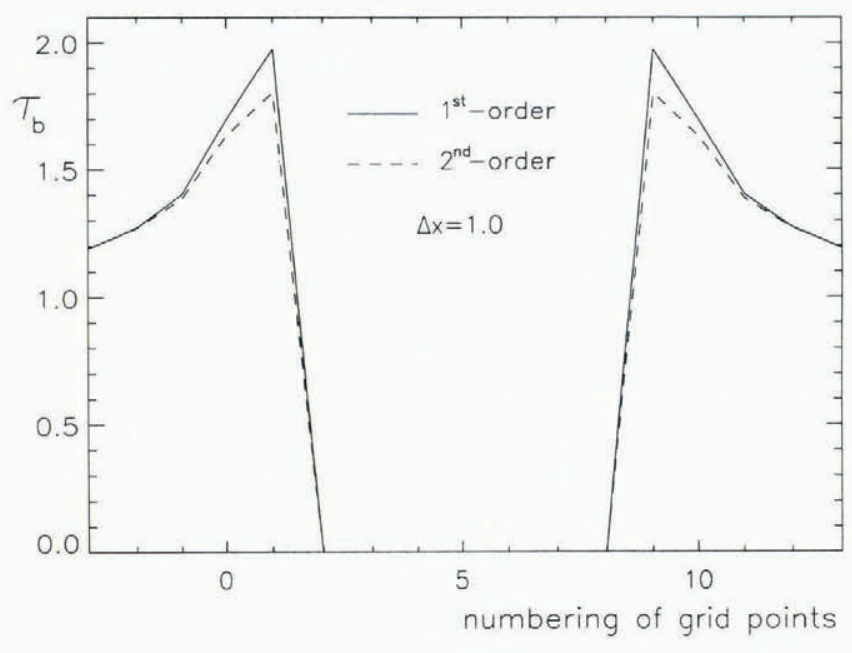

Fig. 1. Longitudinal profiles of basal shear traction in the first-and second-order approximations (terms linear and quadratically in $\epsilon$ are accounted for) across the transitions between non-sliding areas and a limited zone of perfect sliding for a parallel-sided ice slab. The sliding is specified for seven gridpoints $(2-8)$ by prescribed vanishing basal shear traction. The non-sliding parts are defined by vanishing basal velocity. The grid size is $\Delta x=1$ which corresponds to the thickness of the slab.

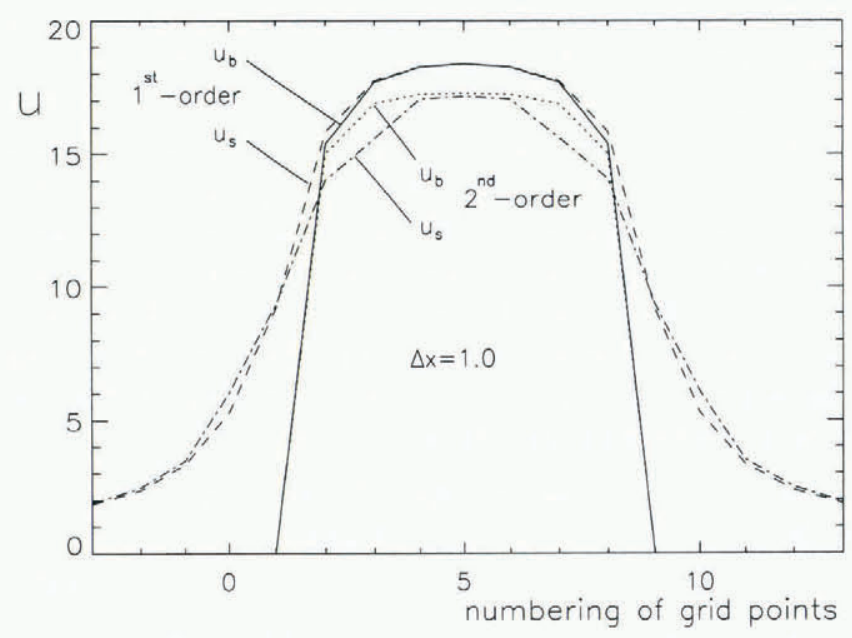

Fig. 2. Longitudinal profiles of basal and surface longitudinal velocity component in the first-and second-order approximations across the transitions between non-sliding areas and a limited zone of perfect sliding as in Figure 1. The subscripts $\mathrm{s}$ and $\mathrm{b}$ refer to surface and base, respectively, the solid and dashed lines represent first-order solutions and the dotted and dash-dotted lines the second-order solutions.

tionary stress and velocity fields with sharp basal transitions for a given geometry. Such transitions may be unrealistically abrupt (Hutter and Olunloyo, 1980, 1981; Barcilon and MacAyeal, 1993) and, in reality, ice may break at the peak stress locations. On the other hand, this illustrates the extent to which the first-order solution captures the essential patterns of stress-strain relations and produces reliable results relatively close to the second-order solutions.

The second-order solutions show profiles with negative shear stress in the interior of the sliding area (Fig. 5). It is not exactly clear whether this is physically realistic or whether it is a numerical artefact, or both. In the solution for $\tilde{\tau}$, this looks like a small oscillation around zero-stress values with a wavelength of two gridcells (Fig. 6). This fact 


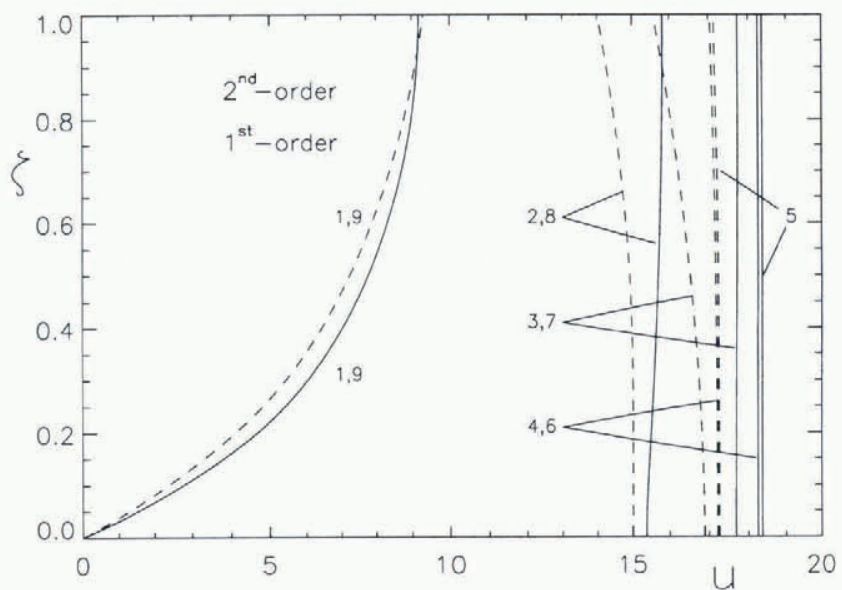

Fig. 3. Vertical profiles of longitudinal velocity inside and adjacent to an area of perfect sliding as described in Figure 1. The labels refer to the corresponding gridpoints as illustrated in Figure 1. The decrease in velocity with distance to the bed is assumed to be a numerical artefact in the second-order solutions.

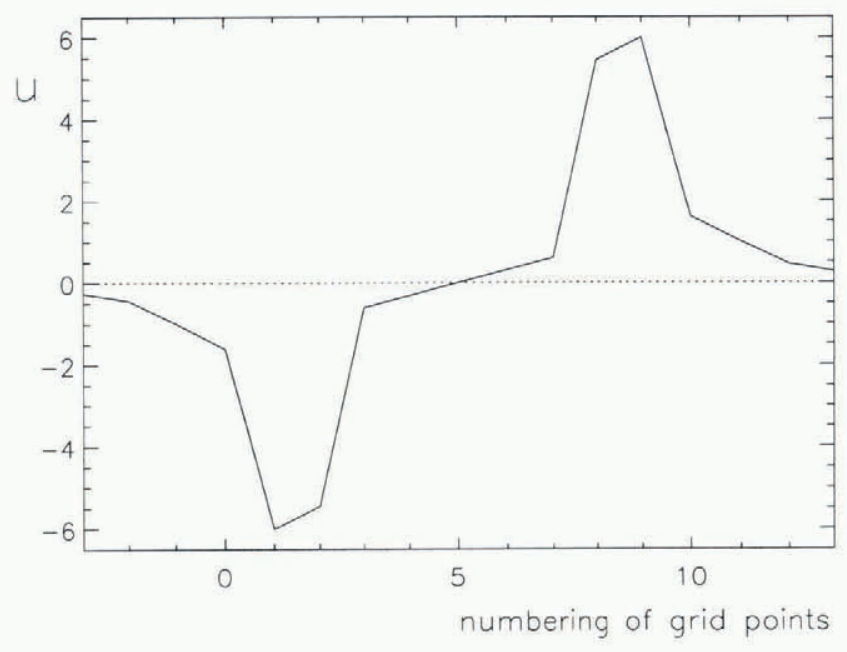

Fig. 4. Longitudinal profiles of vertical velocity component at the surface in the second-order approximation across the transitions between non-sliding areas and a limited zone of perfect sliding as in Figure 1.

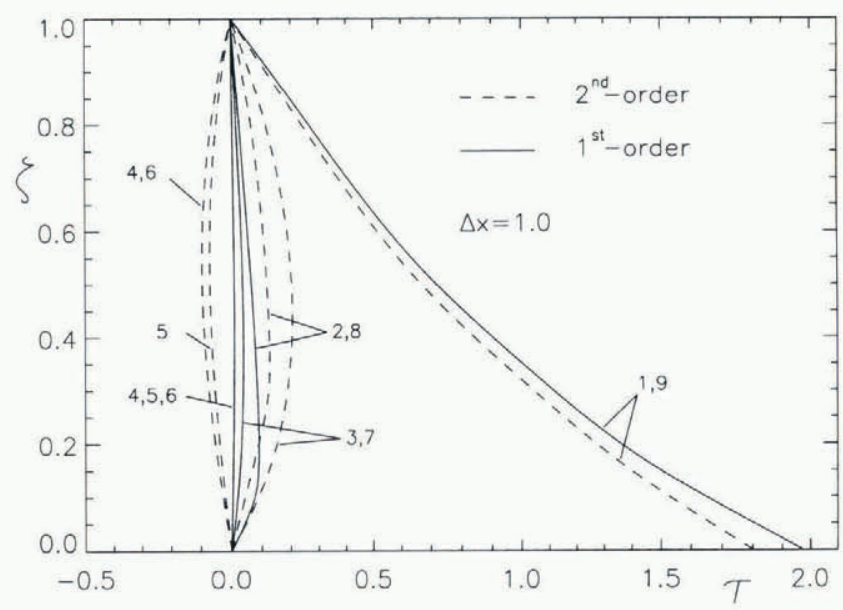

Fig. 5. Vertical profiles of shear stress inside and adjacent to an area of perfect sliding as described in Figure 1. The labels refer to the corresponding gridpoints as illustrated in Figure 1. The negative shear-stress profiles are assumed to be a numerical artefact in the second-order solutions.

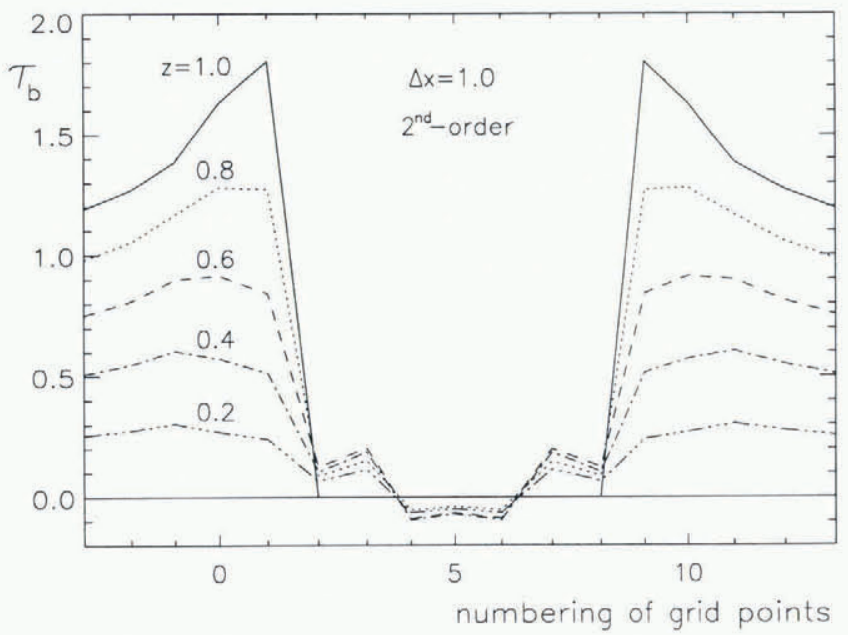

Fig. 6. Longitudinal profiles of shear stress at various levels of $z$ in the second-order approximation across the transitions between non-sliding areas and a limited zone of perfect sliding as in Figure 1. The negative shear stresses are assumed to be a numerical artefact in the second-order solutions.

strongly indicates that the oscillation is numerically induced, although the exact reason is not clear. The problem stems from the $T$ term (see Equation (20) and thus far no solution is available.

\section{CONCLUSIONS AND PROSPECTS}

The governing equations for the two-dimensional stress and velocity fields in glaciers are presented in Cartesian and terrain-following coordinates. A scaling analysis allows the definition of a hierarchy of approximations based on powers of the small-aspect ratio of the ice geometry. Deleting terms that contain $\epsilon$ to powers 2 and higher leads to a set of three coupled non-linear partial differential equations, referred to as first-order approximation in this paper. We present discretization schemes based on the method of lines and discuss various integration and iteration schemes.

The single-shooting fixed-point iteration is easy to program and, for large ice masses with small aspect ratios, it converges reasonably fast (Blatter, 1995). At present, this is the method of choice for three-dimensional finite-difference modelling of higher-order glacier mechanics and dynamics. In this paper, we introduce two-dimensional (plane-strain) models with more elaborate numerical methods: Newton iteration instead of fixed-point iteration and multiple shooting instead of single shooting.

The Newton iteration scheme is faster than the fixedpoint scheme for the same glacier and the same horizontal resolution $\Delta x$. For large ice sheets with smooth surface topography, the Newton iteration allows for substantially smaller $\Delta x$ than the fixed-point iteration. For small glaciers with smooth topography, this improvement is only small but, for glaciers with steep sections and variable surface profile, the fixed-point iteration performed better. Fixedpoint iteration only works reasonably well, if basal velocity is prescribed along the entire bed. On the other hand, Newton iteration can adequately handle mixed basal conditions, where the velocity is prescribed only at parts of the bed and basal shear traction at the rest of the bed. This allows the modelling of glaciers with floating parts such as on subglacial lakes or ice shelves. 
The multiple-shooting scheme allows much better spatial resolution than the single-shooting scheme, with $\Delta x$ as small as $5 \%$ of the average ice thickness for the first-order approximation. However, this is at the price of long computation times and large work-memory requirements. An application, which corroborates these statements is presented in Paper II. This scheme may still be practical, if single situations for the stress and velocity fields are to be modelled. If the mechanical model must be used repeatedly for coupling a surface-evolution model, the single shooting with a well-implemented Newton iteration is certainly the method of choice. In the second-order approximation, the $T$ term must be iterated parallel to the Newton iteration. This reduces the convergence radius and, thus far, the best horizontal resolution that could be achieved with the second-order code was about $75 \%$ of the average ice thickness. More elaborate discretization schemes, which promise faster computation with higher spatial resolution suitable for threedimensional ice masses, are presently being investigated.

The physics that is included in the glacier model presented here poses a novel problem concerning the required information on the glacier to exploit fully the capabilities of the model. The bed topography becomes available for a growing number of glaciers. The general lack of information on sliding patterns poses the most serious limitation, since the longitudinal coupling of stresses strongly depends on the spatial variations of the basal velocity; see Paper II. On the other hand, these requirements point to the desirable field measurements that provide the necessary input for their subsequent interpretation based on model computation (Harbor and others, 1997; Hubbard and others, 1998).

\section{ACKNOWLEDGEMENTS}

We thank A. Ohmura and G. Wanner for their support and stimulating discussions. K. Hutter commented on an earlier version of this paper and helped to improve the manuscript substantially.

\section{REFERENGES}

Ascher, U. M., R. M. Matheij and R. D. Russell. 1988. Numerical solution of boundary value problems for ordinary differential equations. Engelwood Cliffs, NJ, Prentice-Hall. Series in Computational Mathematics.
Bahr, D. B., W. T. Pfeffer and M. F. Meier. 1994. Theoretical limitations to englacial velocity calculations. f. Glaciol., 40 (136), 509-518.

Barcilon, V. and D. R. MacAyeal. 1993. Steady flow of a viscous ice stream across a no-slip/free-slip transition at the bed. F. Glaciol., 39 (131), 167-185.

Blatter, H. 1995. Velocity and stress fields in grounded glaciers: a simple algorithm for including deviatoric stress gradients. 7 . Glaciol., 41 (138), 333-344.

Blatter, H., G. K. C. Clarke andJ. Colinge. 1998. Stress and velocity fields in glaciers: Part II. Sliding and basal stress distribution. J. Glaciol., 44(148), $457-466$.

Dahl-Jensen, D. 1989. Steady thermomechanical flow along two-dimensional flow lines in large grounded ice sheets. f. Geophys. Res., 94 (B8), $10,355-10,362$.

Harbor, J., M. Sharp, L. Copland, B. Hubbard, P. Nienow and D. Mair. 1997. The influence of subglacial drainage conditions on the velocity distribution within a glacier cross section. Geology), $25(8), 739-742$.

Herterich, K. 1987. On the flow within the transition zone between ice sheet and ice shelf. In Van der Veen, C. J. and J. Oerlemans, eds. Dynamics of the West Antarctic ice sheet. Dordrecht, etc., D. Reidel Publishing Co., 185- 202.

Hubbard, A., H. Blatter, P. Nienow, D. Mair and B. Hubbard. 1998. Comparison of the first order approximation for glacier flow with field data: Haut Glacier d'Arolla, Switzerland. f. Glaciol., 44(147), 368-378.

Hutter, K. 1981. The effect of longitudinal strain on the shear stress of an ice sheet: in defence of using stretched coordinates. F. Glaciol., 27 (95), 39-56.

Hutter, K. 1983. Theoretical glaciology; material science of ice and the mechanics of glaciers and ice sheets. Dordrecht, etc., D. Reidel Publishing Co.; Tokyo, Terra Scientific Publishing Co.

Hutter, K. and V. O. S. Olunloyo. 1980. On the distribution of stress and velocity in an ice strip, which is partly sliding over and partly adhering to its bed, by using a Newtonian viscous approximation. Proc. R. Soc. London, Ser. A, 373 (1754), 385-403.

Hutter, K. and V. O. S. Olunloyo. 1981. Basal stress concentrations due to abrupt changes in boundary conditions: a cause for high till concentration at the bottom of a glacier. Ann. Glaciol., 2, 2933.

Lliboutry, L. A. 1987. Very slow flows of solids: basics of modeling in geodynamics and glaciology. Dordrecht, etc., Martinus Nijhoff Publishers.

Muller, H. C. 1991. Une méthode iterative simple pour résoudre les équations de mouvement d'un glacier. (Mémoire de diplôme en mathématique, Université de Genéve. Section de Mathématique.)

Reeh, N. 1988. A flow-line model for calculating the surface profile and the velocity, strain-rate, and stress fields in an ice sheet. F. Glaciol., 34(116), 46-54.

Smith, G. D. and L. W. Morland. 1981. Viscous relations for the steady creep of polycrystalline ice. Cold Reg. Sci. Technol., 5 (2), 141-150.

Van der Veen, C.J. 1989. A numerical scheme for calculating stresses and strain rates in glaciers. Math. Geol., 21 (3), 363-377.

Van der Veen, C. J. and I. M. Whillans. 1989. Force budget: I. Theory and numerical methods. f. Glaciol., 35(119), 53-60.

Verwer, J. G. and J. M. Sanz-Serna. 1984. Convergence of method of lines approximations to partial differential equations. Computing, 33, 297-313.

Whillans, I. M. 1987. Force budget of ice sheets. In Van der Veen, C. J. and J. Oerlemans, eds. Dynamics of the West Antarctic ice sheet. Dordrecht, etc., D. Reidel Publishing Co., 17-36. 